\title{
A Potential Solution for Easing Authentication of Respondents for a Web-Based Survey?
}

Herbert Baum *

Tags: survey practice

\section{Survey Practice}

Vol. 5, Issue 1, 2012

A Potential Solution for Easing Authentication of Respondents for a Web-Based Survey?

While multiple responses may be acceptable for American Idol, serious survey research dictates that we try to prevent people from responding multiple times to a survey. In addition, when asking about specific events we need to ensure that the correct individual is responding. There is an increasing use of Web-based surveys for both of these activities. Survey researchers are increasingly using multi-mode surveys, one of which is Web-based, to increase response rates; and corporations are using Web surveys as an inexpensive way for obtaining consumer feedback. Both these applications require authenticating the respondent to ensure that they have not responded previously to the survey or that they have obtained the service.

Typically, authentication is accomplished by assigning each person a unique identification number that is used by that individual when responding to the survey. This, however, may actually deter people from responding. Two personal examples are listed below:

1. The Red Cross sent me a card requesting that I provide feedback on my experience of donating blood. I decided to comply and went to their Website. After a few clicks I arrived at their customer satisfaction survey. The first question asked me to provide my donor ID number. That is not something I know or have readily accessible, so I did not complete the survey.

2. My brokerage company requested that I complete a survey on my satisfaction with my broker. It was a multi-mode survey (paper and Web). To respond via the Web required an 11 digit survey ID and a six digit access code. Both of these appeared to be randomly generated.

DRC has its own experience with a multi-mode survey conducted for the 
Maryland State Department of Education which requires that the contractor establish a mechanism to prevent a person from responding multiple times. We did this by assigning each person a unique identifier. There were over 10,000 respondents and less than $5 \%$ responded via the Web.

\section{WHAT IS THE SOLUTION?}

Rather than assigning a number that respondents cannot remember, why not ask the respondents to supply information that may uniquely identify them? However, selecting a unique identifier is not a trivial task. These three examples that come readily to mind are not unique:

1. Cell phones are ubiquitous, but a cell phone number, for example, can be shared between two or more individuals.

2. Birthdates are not unique, and if you have 367 or more randomly chosen people, there is a $100 \%$ probability that at least one pair of them will have the same birthday (Wikipedia).

3. Federal contracts often prohibit us from gathering e-mail addresses, but often an individual will have multiple addresses (e.g., work and home).

The challenge comes in finding the least number of criteria that the respondent is likely to know that uniquely identifies them and that represents information they are willing to share.

I would have gladly responded to the Red Cross if they asked me the following:

1. In which state did you make your last blood donation?

2. In which city within that state did you make that donation?

3. What is your birth date?

4. What is your first name?

This is all information I know and should uniquely identify me.

What if it does not uniquely identify me? An error message could appear indicating that there are multiple records with these characteristics; please call 1-800-xxx-HELP. If the authentification worked $50 \%$ of the time, the response rates would be much higher and very few of the remaining $50 \%$ are likely to call.

What do you do if somebody calls? I recommend that your database be accessible to a few key individuals and that calls be routed to them. Those individuals could then find the unique record by asking one or two additional questions. Once the record is identified, I suggest completing the survey via the telephone by entering the information directly into the database. 


\section{CONCLUSION}

Though the author of this quote is unknown, it has been used to define insanity: "Doing the same thing over and over again and expecting different results." If we continue providing potential Web survey respondents with unique identifiers that do not make sense to them, we will continue to have low response rates. This brief piece poses that response rates can be increased by letting the respondent answer a series of questions about themselves that uniquely identifies them and contains information they would be willing to share. The challenge for survey researchers is developing for each study that minimal list of questions. 